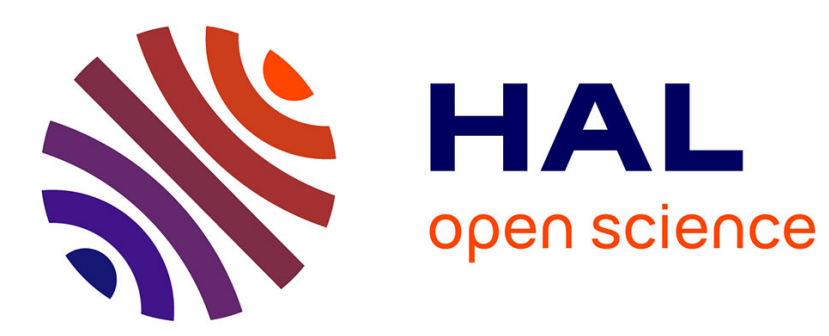

\title{
Simulations of Biomolecule Unbinding from Protein Using DL_POLY \\ P-L Chau
}

\section{To cite this version:}

P-L Chau. Simulations of Biomolecule Unbinding from Protein Using DL_POLY. Molecular Simulation, 2007, 32 (12-13), pp.953-961. 10.1080/08927020600835640 . hal-00514985

\section{HAL Id: hal-00514985 \\ https://hal.science/hal-00514985}

Submitted on 4 Sep 2010

HAL is a multi-disciplinary open access archive for the deposit and dissemination of scientific research documents, whether they are published or not. The documents may come from teaching and research institutions in France or abroad, or from public or private research centers.
L'archive ouverte pluridisciplinaire HAL, est destinée au dépôt et à la diffusion de documents scientifiques de niveau recherche, publiés ou non, émanant des établissements d'enseignement et de recherche français ou étrangers, des laboratoires publics ou privés. 


\section{Molecular Simulation \\ Journal of \\ Experimental Nanoscience \\ - Taylor \& Francis \\ Taglor \& Francis Group}

\section{Simulations of Biomolecule Unbinding from Protein Using DL_POLY}

\begin{tabular}{|r|l|}
\hline Journal: & Molecular Simulation/Journal of Experimental Nanoscience \\
\hline Manuscript ID: & GMOS-2006-0030.R1 \\
\hline Journal: & Molecular Simulation \\
\hline Date Submitted by the \\
Author: & $10-$ May-2006 \\
\hline Complete List of Authors: & Chau, P-L; Institut Pasteur, Bioinformatique Structurale \\
\hline Keywords: & $\begin{array}{l}\text { molecular dynamics, molecular recognition, free energy calculation, } \\
\text { serotonin, retinol }\end{array}$ \\
\hline
\end{tabular}

\section{SCHOLARONE Manuscripts}




\title{
Simulations of Biomolecule Unbinding from Protein Using DL_POLY
}

\author{
P.-L. Chau \\ Bioinformatique Structurale \\ CNRS URA 2185 \\ Institut Pasteur \\ 75724 Paris \\ France \\ email : pc104@pasteur.fr
}

\begin{abstract}
This paper briefly reviews the ideas leading to ligand-receptor interaction being a central topic of research in the biological sciences, especially in pharmacology. The simulation methods for studying ligand-receptor interaction dynamically through ligand unbinding are reviewed, together with the analysis methods devised to examine the unbinding trajectory. Examples of applying DL_POLY in these simulations are given; they include retinol unbinding from the retinol-binding protein, and of serotonin and granisetron unbinding from the $5-\mathrm{HT}_{3}$ receptor.
\end{abstract}

Keywords : ligand-receptor interaction, molecular recognition, molecular dynamics, free energy calculations, retinol, retinol-binding protein, serotonin, granisetron, $5-\mathrm{HT}_{3}$ receptor

\section{Biomolecular Interactions}

Biomolecular interaction is the first step to many basic processes of life. Whether it be enzyme catalysis, neurotransmitter and hormone action or antibody-antigen recognition, these processes always involve the interaction of biological molecules 
with each other. The importance of molecular interaction has been gradually realised over history through pharmacological and chemical experiments.

Many pharmacology experiments have been performed since time immemorial, and many valuable discoveries have been made, and the results were compiled systematically in various publications. The first Chinese pharmacopeia is the Shen Nong Ben Cao Jing of Han Dynasty (206 B.C.-220 A.D.), which contains descriptions of over 300 substances of pharmacological importance ${ }^{1}$. Dioscorides Pedanius' De materia medica ${ }^{2}$ is the first pharmacopeia to appear in Europe during the 1st century A.D., and contains description of 600 plant products and 70 animal products. Nevertheless, no effort was made to construct a unifying theory that underlies the observations.

At the same time, advances in chemistry enabled pharmacologists to separate drugs into different parts. Scientists discovered that the effect of many drugs was due to an active principle. For example, Sertürner isolated morphine and meconic acid from opium in 1803, and demonstrated that the sedative property of opium was due exclusively to morphine (which he called "morphium") ${ }^{3}$; this was achieved by testing morphine on his friends.

Paul Ehrlich ${ }^{4}$ formulated the Seitenketten-Theorie to account for the interaction between drugs and receptors: biological tissues contained side-chains, which bound toxins or dyes. The original function of the side-chains was not to bind toxins or dyes, but to carry out certain essential physiological functions. Different tissues had different side-chains, and since dyes had different affinities for these side-chains, the staining of vital dyes was specific. Toxins were harmful because they bound to the side-chains on the tissues, preventing the side-chains from carrying out their physiological functions. Antitoxins bound to the toxins to prevent the latter from coming into contact with side-chains on the tissues.

Ehrlich's conclusions spurred scientists on to uncover links between the

\footnotetext{
${ }^{1}$ English translation by S.-Z. Yang (1998) Divine Farmer's Materia Medica, Blue Poppy Press, Boulder, Colorado, U.S.A.

${ }^{2}$ Dioscorides Pedanius of Anazarbos, De materia medica, 1518 edition published in Venice by Aldus Manutius.

${ }^{3}$ F.W.A. Sertürner (1817) Über das Morphium, eine neue salzfähige Grundlage, und die Mekonsäure, als Hauptbestandtheile des Opium, Annalen der Physik, 55, 56-90.

${ }^{4}$ P. Ehrlich (1909) Über den jetzigen Stand der Chemotherapie, Berichte der Deutschen Chemischen Gesellschaft, 42, 17-47.
} 
chemical structure of drugs and their biological effects. Pharmacologists came to realise that a change in the chemical structure could bring about a change in the biological activity. The desired biological activity could be amplified and the unwanted activity diminished or abolished by appropriate chemical modifications of pre-existing ligands. A ligand is a small molecule that binds to the receptor site in question. It can be a drug, a natural hormone or neurotransmitter. Following Ehrlich's nomenclature, the molecule inside the body is called the receptor, and the introduced molecule is called the ligand. New drugs were discovered by systematic alteration of these ligands.

In the course of this work, it was realised that a better understanding of the detailed atomic structure of the receptor would facilitate our understanding. Since most biological receptors are proteins, this has to wait for the development of crystallography ${ }^{5}$ and resolution of the structure of the proteins. The first protein thus studied, haemoglobin, also happens to bind a simple ligand, the oxygen molecule ${ }^{6}$. From then on, many soluble protein receptors have been crystallised, and their atomic structure resolved. Membrane protein receptors have to wait till the development of electron microscopy image processing techniques ${ }^{7}$ before their structures were resolved ${ }^{8}$. In addition, nuclear magnetic resonance methods can also be used to solve the structures of proteins ${ }^{9}$.

Crystallography, electron microscopy, and nuclear magnetic resonance are invaluable in elucidating the structure of proteins. Nevertheless, they do not provide us with simultaneous information about the structure and dynamics of the ligand-

\footnotetext{
${ }^{5}$ W.H. Bragg (1913) The reflection of X-ray by crystals, Proceedings of the Royal Society, London, 89A, 246-248; W.L. Bragg (1913) The structure of some crystals as indicated by their diffraction of X-rays, Proceedings of the Royal Society, London, 89A, 248-277.

${ }^{6}$ M.F. Perutz (1942) Crystal structure of oxyhaemoglobin, Nature, 150, 324-329.

${ }^{7}$ W.O. Saxton (1978) Computer Techniques for Image Processing in Electron Microscopy, supplement to volume 10 of Advances in Electronics and Electron Physics series, Academic Press, New York.

${ }^{8} \mathrm{~N}$. Unwin (1993) Nicotinic acetylcholine receptor at $9 \AA$ resolution, Journal of Molecular Biology, 229, 1101-1124; N. Unwin (1995) Acetylcholine receptor channel imaged in the open state, Nature, 373, 37-43; A. Miyazawa, Y. Fujiyoshi and N. Unwin (2003) Structure and gating mechanism of the acetylcholine receptor pore, Nature, 423, 949-955; Nigel Unwin (2005) Refined structure of the nicotinic acetylcholine receptor at $4 \AA$ resolution, Journal of Molecular Biology, 346, 967-989.

${ }^{9}$ R. Kaptein, E.R.P. Zuiderweg, R.M. Scheek, R. Boelens, R., and W.F. van Gunsteren (1985) A protein structure from NMR data. lac repressor headpiece, Journal of Molecular Biology, 182, 179-182; M.P. Williamson, T.F. Havel and K. Wüthrich (1985) Solution conformation of proteinase inhibitor IIA from bull seminal plasma by ${ }^{1} \mathrm{H}$ nuclear magnetic resonance and distance geometry, Journal of Molecular Biology, 182, 295-315.
} 
receptor complex and the solvent molecules, crucial to understanding the binding process. Furthermore, it is difficult to isolate the factors that contribute to binding, and quantify their relative importances in each case. Simulations complement theory and experiments by making it possible to study each factor in depth and to obtain structural and dynamical details simultaneously at picosecond resolutions.

It is known that water mediates the interaction between ligands and receptors. This could be done via steric effects ${ }^{10}$, bridging hydrogen bonds ${ }^{11}$ or entropic effects ${ }^{12}$. The effect of water is very important and it can radically change the interaction between ligands and receptors. Solvation and interaction of biological molecules is a complex many-bodied problem, and there exist no analytical methods for such investigations; only simulation methods, such as molecular dynamics (MD) and Monte Carlo (MC) methods, can be used.

\section{Non-dissociatve Studies of Biomolecular Complexes}

The earliest simulations on ligand-receptor complexes are all 'non-dissociative' simulations. 'Non-dissociative' means that no attempt has been made to dissociate the ligand from the receptor in these simulation studies.

Åqvist et al. ${ }^{13}$ performed the first molecular dynamics simulation on a holoprotein / apo-protein pair to study the effect of ligand binding on protein dynamics. They took the experimental coordinates of holo-serum retinol-binding protein, and used the GROMOS potential for a molecular dynamics simulation of $70 \mathrm{ps}$. They did not have the experimental coordinates of the apo-protein, so

\footnotetext{
${ }^{10}$ C.S. Poornima and P.M. Dean (1995) Hydration in drug design. 1. Multiple hydrogenbonding features of water molecules in mediating protein-ligand interactions, Journal of Computer-Aided Molecular Design, 9, 500-512; D.S. Wilson, B. Guenther, C. Desplan and J. Kuriyan (1995) High resolution crystal structure of a paired (Pax) class cooperative homeodomain dimer on DNA, Cell, 82, 709-719.

${ }^{11}$ B.C. Braden, B.A. Fields and R.J. Poljak (1995) Conservation of water molecules in an antibody-antigen interaction, Journal of Molecular Recognition, 8, 317-325.

${ }^{12}$ R. de Cristofaro, M. Picozzi, E. de Candia, B. Rocca and R. Landolfi (1995) Thrombinthrombomodulin interaction - energetics and potential role of water as an allosteric effector, Biochemical Journal, 310, 49-53; D.N. Dubins, R. Filfil, R.B. MacGregor and T.V. Chalikian (2000) Role of water in protein-ligand interactions: volumetric characterisation of the binding of 2'-CMP and 3'-CMP to ribonuclease A, Journal of Physical Chemistry B, 104, 390-401.

${ }^{13}$ J. Åqvist, P. Sandblom, T.A. Jones, M.E. Newcomer, W.F. van Gunsteren and O. Tapia (1986) Molecular dynamics simulations of the holo and apo forms of retinol binding protein, Journal of Molecular Biology, 192, 593-604.
} 
extracted the protein coordinates from the holo-protein experimental coordinates, and used that as the initial point of an 80-ps simulation of the apo-protein. Both simulations were performed in vacuum at $298 \mathrm{~K}$ using an NVT ensemble, where the number of atoms, the volume of the simulation box and the temperature were held constant, and the first 30 ps was used for equilibration. In their holo-protein simulation, they discovered that the root-mean-square deviation of the simulation coordinates from the experimental coordinates is $3.0 \AA$ for all atoms, and $2.2 \AA$ for $\mathrm{C}_{\alpha}$-atoms only.

Tilton et al. studied the myoglobin-xenon complex ${ }^{14}$ using simulations with the AMBER potential. In their 96-ps simulations, they established that the r.m.s. difference between the X-ray experimental structure and their simulated structure was $1.25 \AA$, and that the volume of the simulated structure oscillated at 3-4\% around the X-ray volume. Of the five xenon atoms placed in myoglobin, three of them moved by less than $3.5 \AA$. The other two both moved by about $10 \AA$; one escaped to the surface of the protein, and the other went into the hydrophobic core.

Dahl et al. created a homology model of the dopamine $\mathrm{D}_{2}$ receptor, pulled dopamine into the binding site in 80 ps using docking molecular dynamics, and performed a minimisation of the ligand inside the binding site $\mathrm{e}^{15}$. Unfortunately the details of the docking procedure were not mentioned, and the authors only reported that dopamine was in close proximity to Asp 80 and Asn 390.

Non-dissociative studies aimed to validate the simulations with experimental data, by comparing the simulated structure with the crystallographic or NMR structure. There were some attempts to locate the interactions between the ligand and the receptor. Subsequently, as computer power improved, attempts were made to simulate the unbinding of ligands from receptors.

\footnotetext{
${ }^{14}$ R.F. Tilton, U.C. Singh, I.D. Kuntz and P.A. Kollman (1988) Protein-ligand dynamics: a 96-ps simulation of a myoglobin-xenon complex, Journal of Molecular Biology, 199, 195-211.

${ }^{15}$ S.G. Dahl, Ø. Edvardsen and I. Sylte (1991) Molecular dynamics of dopamine at the $\mathrm{D}_{2^{-}}$ receptor, Proceedings of the National Academy of Sciences, USA, 88, 8111-8115.
} 


\section{Methods for Unbinding Ligands from Recep- tors}

\subsection{Previous work - steered molecular dynamics}

Steered molecular dynamics (SMD) was developed to induce conformational changes in proteins, but was later used to simulate ligand unbinding from the receptor ${ }^{16}$. In this method, potential-well restraints were added to part of the protein; these restraints were external forces which behaved like a spring with one end fixed to the target position, and the other end attached to the atom being "tugged". In their seminal paper, Leech et al. discussed the uses of the method, and also pointed out that faster and stronger "tugs" could cause disordering effects on the system, and that the only way to verify that the pathway was physically correct was to do experiments. Grubmüller et al. applied this method to perform simulations of the unbinding of the streptavidin-biotin complex ${ }^{17}$. The authors obtained the unbinding trajectory from previous experiments, and were able to reproduce experimental data using simulation methods. They used a number of unbinding speeds, ranging from $40 \mathrm{~m} / \mathrm{s}$ to $0.15 \mathrm{~m} / \mathrm{s}$, and noted that the rupture force is velocity dependent, as expected, up to $1.5 \mathrm{~m} / \mathrm{s}$. Thereafter the rupture force saturates, though they still observed a scatter in the value of the rupture force. They attributed this to the heterogeneity of unbinding pathways, arising from the microheterogeneity of protein conformational substates. They identified conformational changes of biotin during the unbinding process, and the breaking of hydrogen bonds between the ligand, the receptor and some surrounding water molecules.

The SMD method has been applied to the avidin-biotin complex ${ }^{18}$ in simulations of length $40-500 \mathrm{ps}$. The authors applied a harmonic force constant close to those of atomic force microscopy (AFM) magnitudes to pull biotin out from avidin. They found that the calculate rupture forces were much larger than those observed in experiments. They concluded that, in these short simulations, the

\footnotetext{
${ }^{16}$ J. Leech, J. Prins and J. Hermans (1996) SMD: visual steering of molecular dynamics for protein design, IEEE Computational Science and Engineering, 3, 38-45.

${ }^{17}$ H. Grubmüller, B. Heymann and P. Tavan (1996) Ligand binding: molecular mechanics calculation of the streptavidin-biotin rupture force, Science, 271, 997-999.

${ }^{18}$ S. Izrailev, S. Stepaniants, M. Balsera, Y. Oono and K. Schulten (1997) Molecular dynamics study of unbinding of the avidin-biotin complex, Biophysical Journal, 72, 1568-1581.
} 
rupture forces required were much stronger than those used in millisecond AFM experiments, so the simulated unbinding cannot be extrapolated to experimental results.

Subsequent work focused on the unbinding of retinoic acid from the retionic acid receptor ${ }^{19}$. Since the authors did not know the pathway(s) of unbinding, they applied three unbinding pathways, using an unbinding speed of $0.032 \AA / p s$ $(3.2 \mathrm{~m} / \mathrm{s})$. They concluded that pathway 2 involved large changes in protein conformation, while pathways 1 and 3 disturbed protein conformation to a lesser extent. Using structural arguments, they suggested that pathway 1 was the binding pathway, while pathway 3 was the unbinding pathway. However, no attempt was made to verify this experimentally.

A combined simulation and crystallographic study was performed in the same year $^{20}$. The authors created a D128A mutant of streptavidin, and resolved its structure with and without biotin bound to it. They also performed ten simulations to gradually pull biotin out of the streptavidin binding pocket. A large number of simulations were required to sample the phase space adequately. In wild-type streptavidin, the authors observed that the carboxylate side-chain of Asp 128 made a hydrogen bond with biotin. In the course of the simulated unbinding, this hydrogen bond lengthened and was replaced by a water-mediated contact between Asp 128 and biotin. In the case of the D128A mutant, a water molecule was observed between biotin and Ala 128 even in the initial stages. Thermodynamic analysis showed that the D128A mutant behaved like an intermediate in the dissociation process. This work showed that, given enough dissociation pathways, SMD simulations could provide insight into the unbinding process.

Heymann and Grubmüller ${ }^{21}$ performed a series of SMD simulations on a complex formed by dinitrophenyl (DNP) and the monoclonal antibody AN02 $\mathrm{F}_{\mathrm{ab}}$ fragment. Some of these simulations had different starting conditions, while in

\footnotetext{
${ }^{19}$ D. Kosztin, S. Izrailev and K. Schulten (1999) Unbinding of retinoic acid from its receptor studied by steered molecular dynamics, Biophysical Journal, 76, 188-197.

${ }^{20}$ S. Freitag, V. Chu, J.E. Penzotti, L.A. Klumb, R. To, D. Hyre, I. le Trong, T.P. Lybrand, R.E. Stenkamp and P.S. Stayton (1999) A structural snapshot of an intermediate on the streptavidin-biotin dissociation pathway, Proceedings of the National Academy of Sciences, USA, 96, 8384-8389.

${ }^{21} \mathrm{~B}$. Heymann and H. Grubmüller (2001) Molecular dynamics force probe simulations of antibody/antigen unbinding: entropic control and non-additivity of unbinding forces, Biophysical Journal, 81, 1295-1313.
} 
others, the pulling speed were assigned values from $0.1 \mathrm{~m} / \mathrm{s}$ and $50 \mathrm{~m} / \mathrm{s}$. They identified two main events leading to DNP unbinding from AN02 $\mathrm{F}_{\mathrm{ab}}$ : rupture of hydrogen bond between Tyr 33 of the light chain, and opneing up of the tryptophane sandwich. They were able to identify two broad classes of unbinding pathways: in the 'light chain route', the tryptophane sandwich opened first, while in the 'heavy chain route', the hydrogen bond ruptured first.

Bayas et al. ${ }^{22}$ applied SMD to unbind CD2 from CD58. They found that at high pulling speeds of $0.01 \mathrm{~m} / \mathrm{s}$ and $5 \times 10^{-3} \mathrm{~m} / \mathrm{s}$, the two proteins unfolded before separation. At lower unbinding speeds of $10^{-3} \mathrm{~m} / \mathrm{s}$ and $5 \times 10^{-4} \mathrm{~m} / \mathrm{s}$, the proteins separated before the domains could unfold. This work showed the importance of using a more realistic unbinding speed in SMD so as not to perturb the protein structure.

Whilst the SMD method has seen wide use in simulation studies of ligandprotein interactions, it does suffer from the drawback that the unbinding trajectory has to be pre-defined. When this is not known, scientists have to use a number of alternative trajectories to decide which ones are more probable. To overcome this problem, a new method do not require the prior definition of the unbinding pathway has recently been developed.

\subsection{Mutual repulsion}

In the method of mutual repulsion ${ }^{23}$, the centres of mass of the ligand and the receptor are assigned what can be called "pseudo-charges", $g$, that increase linearly with time. A potential $\Psi(\mathbf{r})$ is defined:

$$
\Psi\left(\mathbf{r}_{i}\right)=\frac{g^{2}}{\left|\mathbf{R}_{1}-\mathbf{R}_{2}\right|}
$$

where $\mathbf{r}_{i}$ is the position vector of the atom $i, g$ is the magnitude of the pseudocharge at that time, $\mathbf{R}_{1}$ is the position vector of the centre of mass of the receptor, and $\mathbf{R}_{2}$ is that of the centre of mass of the ligand.

The pseudo-charges interact with each other, but do not affect the normal electronic partial charges assigned to each atom. They repel or attract each other

\footnotetext{
${ }^{22}$ M.V. Bayas, K. Schulten and D. Leckband (2003) Forced detachment of the CD2-CD58 complex, Biophysical Journal, 84, 2223-2233.

${ }^{23}$ P.-L. Chau (2001) Process and thermodynamics of ligand-receptor interaction studied using a novel simulation method, Chemical Physics Letters, 334, 343-351.
} 
under rules similar to those for normal electronic partial charges. This method approaches the problem of unbinding as a rare event with large energy difference between the bound and the unbound states. The $\Psi\left(\mathbf{r}_{i}\right)$ potential artificially reduces this energy difference so that the transition from one state to another is facilitated.

There are two advantages of the method. Firstly, since the force due to the pseudo-charges is calculated with respect to the centre of mass, no torque is generated on the molecules. The molecules will be allowed to explore the unbinding path with fewer artificial forces. Secondly, since the pseudo-charge increases slowly, the potential $\Psi\left(\mathbf{r}_{i}\right)$ can be exploited for the calculation of the Helmholtz free energy $\Delta A$ by the adiabatic switching method, as explained later.

To demonstrate the validity of this method, I applied it to unbind retinol from the bovine serum retinol-binding protein. Atomic coordinates of the holo-sRBP 24 were obtained from the Protein Databank (PDB code: 1HBP); the structure of the protein is shown in figure 1 . The model for the protein and retinol was parameterised using the GROMOS 87 potential ${ }^{25}$. With conservation of crystallographically determined water molecules, the protein was solvated in truncated octahedral boxes filled with $5537 \mathrm{SPC} / \mathrm{E}$ water ${ }^{26}$ molecules using the wateradd utility in DL_POLY. Four sodium ions were added to ensure total electronic neutrality.

All minimisations and simulations were performed using version 2 of DL_POLY ${ }^{27}$. The solvated structure was minimised using the zero-K minimisation option, and simulations were started with an NVT run at $30 \mathrm{~K}$, taking initial velocities from a Maxwellian distribution. The temperature was gradually increased in steps to $310 \mathrm{~K}$ over a period of $100 \mathrm{ps}$, using a Nosé-Hoover thermostat ${ }^{28}$. SHAKE

\footnotetext{
${ }^{24}$ G. Zanotti, R. Berni, and H. L. Monaco (1993) Crystal-structure of liganded and unliganded forms of bovine plasma retinol-binding protein, Journal of Biological Chemistry, 268, 1072810738.

${ }^{25}$ W.F. van Gunsteren and H.J.C. Berendsen (1987) Groningen Molecular Simulation Library Manual, Biomos B.V., Groningen, Netherlands.

${ }^{26}$ H.J.C. Berendsen, J.R. Grigera and T.P. Straatsma (1987) The missing term in effective pair potentials, Journal of Physical Chemistry, 91, 6269-6271.

${ }^{27}$ T.R. Forester and W. Smith (1996) DL_POLY 2.0 - a general-purpose parallel molecular dynamics package, Journal of Molecular Graphics, 14, 136-141.

${ }^{28}$ S. Nosé (1984) A unified formulation of the constant temperature molecular dynamics methods, Journal of Chemical Physics, 81, 511-519; W.G. Hoover (1985) Canonical dynamics - equilibrium phase space distributions, Physical Review A, 31, 1695-1697.
} 
was used to constrain bond lengths with a tolerance of $10^{-8}$. The time step was $1 \mathrm{fs}$. A cutoff of $10 \AA$ was used, with simple truncation for coulombic and van der Waals interactions. The simulations were then continued for $400 \mathrm{ps}$ in an NPT ensemble using the Nosé-Hoover thermostat and the Melchionna barostat ${ }^{29}$, all thermostats and barostats being options integral to DL_POLY. The last 200 ps of this NPT simulation was used to determine the optimal box size for a pressure of $1.01 \times 10^{5} \mathrm{~Pa}(1 \mathrm{~atm})$. This box size was then used for a subsequent 100 -ps NVT simulation to equilibrate the system, followed by a mutual repulsion run of $1 \mathrm{~ns}$, again in the NVT ensemble. Configurational data were output every 20 fs during the mutual repulsion run.

Pseudo-charges were given dimensionless units. The force due to the pseudocharges between the centres of mass was:

$$
F=\frac{k g^{2}}{\left|\mathbf{R}_{1}-\mathbf{R}_{2}\right|^{2}}
$$

where $F$ was the magnitude of the force between the two pseudo-charges $(k=$ $1.6605402 \times 10^{-33} \mathrm{~kg} \mathrm{~m}^{3} \mathrm{~s}^{-2}$ and is a constant inserted to ensure that the force due to the pseudo-charges is adjusted to the internal units of DL_POLY, and that it is also of comparable magnitude to the other forces). The pseudo-charges were implemented by inserting the commands in the subroutine extnfld.f; extra forces were assigned to the centres of mass of the ligand and the receptor, respectively, and then equally distributed to all atoms.

The mutual repulsion was coupled with the method of adiabatic switching to give an estimate of the free energy change of unbinding. In the NVT ensemble, the NVE Hamiltonian of the physical system, $H_{0}$, is added to the thermostat part of the Nosé-Hoover thermostat, $H^{\prime}$, to give $H_{n}$, a "Nosé conserved quantity" or a "Nosé pseudo-Hamiltonian":

$$
H_{n}=H_{0}+H^{\prime}
$$

The partition function for this extended Nosé pseudo-Hamiltonian can be written as:

$$
Z=\frac{1}{3 N+1} \sqrt{\frac{2 \pi Q}{k T}} e^{E / k T} Z_{c}^{o}
$$

\footnotetext{
${ }^{29}$ S. Melchionna, G. Ciccotti and B.L. Holian (1993) Hoover NPT dynamics for systems varying in size and shape, Molecular Physics, 78, 533-544.
} 
where $Q$ is a parameter that behaves like a mass in the Nosé-Hoover thermostat and acts as a heat source/sink, and $Z_{c}^{o}$ is the partition function of the NVE ensemble for the physical system $\mathbf{H}_{0}$. Under adiabatic change of $\mathbf{H}_{0}$, an equivalent expression exists for the new Hamiltonian $\mathbf{H}_{f}$ and extended Nosé pseudoHamiltonian $\mathbf{H}_{n, f}$ :

$$
Z_{f}=\frac{1}{3 N+1} \sqrt{\frac{2 \pi Q}{k T}} e^{E^{\prime} / k T} Z_{c}^{f}
$$

When the $\Psi\left(\mathbf{r}_{i}\right)$ potential is applied in addition to the thermostat, the gradually increasing mutual repulsion can be viewed as a switching function of the type described by Watanabe and Reinhardt ${ }^{30}$. These authors also showed that the Helmholtz free energy difference, $\Delta A$, is equal to the total energy difference of the extended Nosé conserved quantity (the NVE Hamiltonian plus the thermostat part) under the adiabatic transformation:

$$
Z_{c}^{f}=Z_{c}^{o} e^{-\Delta A / k T}
$$

It is imperative for the mutual repulsive force to increase very slowly, otherwise this method is no longer valid.

In the beginning of the simulation, the pseudo-charge was held at zero for $50 \mathrm{ps}$. At the end of the simulation, when retinol could not go any further without colliding with the other side of the protein through periodic boundary conditions, the pseudo-charge was held at 290.5, and the system equilibrated for $50 \mathrm{ps}$, followed by a data collection period of $50 \mathrm{ps}$. The final and initial 50-ps periods were used for evaluating the change in Helmholtz free energy when the ligand was unbound from the receptor. Let the initial kinetic energy, potential energy and pseudocharge potential energy be, respectively, $K_{i}, V_{i}$ and $\Psi_{i}$, and their final values be, respectively, $K_{f}, V_{f}$ and $\Psi_{f}$. The kinetic energy and potential energy values are derived from the Nosé conserved quantity. The Helmholtz free energy change $\Delta A$ is given by:

$$
\Delta A=K_{f}+V_{f}+\Psi_{f}-K_{i}-V_{i}-\Psi_{i}
$$

These quantities could all be read off the OUTPUT file of DL_POLY, so it was quite straight-forward to compute $\Delta A$.

\footnotetext{
${ }^{30}$ M. Watanabe and W.P. Reinhardt (1990) Direct dynamical calculation of entropy and free energy by adiabatic switching, Physical Review Letters, 65, 3301-3304.
} 


\section{Results from Retinol Unbinding}

Figure 2 shows retinol and retinol-binding protein at the end stage of the unbinding. It can be seen that retinol cleared the binding site at the end, and that the protein structure was largely unchanged. A quantitative analysis ${ }^{31}$ showed that the r.m.s. deviation of the simulated structure from the crystal structure was always below $2.0 \AA$.

\subsection{Free energy changes}

Both my simulation and previous experiments showed that the free energy change was purely entropic. The experiment was performed in the NPT ensemble ${ }^{32}$, and the entropy change $\Delta S=-364 \mathrm{~J} \mathrm{~mol}^{-1} \mathrm{~K}^{-1}$. This value compares favourably with my NVT simulation result of $\Delta S=-580 \pm 30 \mathrm{~J} \mathrm{~mol}^{-1} \mathrm{~K}^{-1}$.

\subsection{Changes in binding site}

I was able to examine the trajectory to define the movement of water and protein atoms in ligand-receptor interactions. I located the atoms of the binding site, and found that they formed a quasi-convex hull. Triangles were constructed from the polyhedron vertices using a convex hull programme ${ }^{33}$. The binding site volume was found to be about $5900 \AA^{3}$ in the initial 500 ps. It then decreased to below $5700 \AA^{3}$ at about $700 \mathrm{ps}$. During the last $300 \mathrm{ps}$, the volume increased to about $5800 \AA^{3}$ and then decreased to about $5700 \AA^{3}$ (figure 3). So there was a net decrease of about $200 \AA^{3}$.

Using a previously developed method $^{34}$, I calculated the volume occupied by retinol, which was about $270 \AA^{3}$. Thus with the departure of retinol from the binding site, and a decrease in binding volume, there is a net increase of about $70 \AA^{3}$ in the space of the binding site. This is about the volume occupied by two or three water molecules. Would water from outside the binding site enter it, to occupy the space vacated?

\footnotetext{
${ }^{31}$ reference 23

${ }^{32}$ N. Noy and Z.J. Xu (1990) Thermodynamic parameters of the binding of retinol to binding proteins and to membranes, Biochemistry, 29, 3888-3892.

${ }^{33}$ K.L.Clarkson, K.Mehlhorn, and R.Seidel (1993) Four results on randomized incremental constructions, Computational Geometry - Theory and Applications, 3, 185-212.

${ }^{34}$ S.L. Chan, E.O. Purisima (1998) Molecular surface generation using marching tetrahedra, Journal of Computational Chemistry, 19, 1268-1277.
} 


\subsection{Water movements}

The number of water molecules inside the binding site during the unbinding simulation is shown in figure 4. During the first $200 \mathrm{ps}$, there were about 77 water molecules inside the binding site. Over time, the number of water molecules decreased to about 73 . Thus, at least within the timescale of the simulation, there was net loss of water from the binding site, which is surprising ${ }^{35}$. How would the water molecules re-arrange themselves to accommodate the changes?

A detailed analysis was carried out to examine the trajectory of each individual water molecule. I calculated the actual and net distances traversed by each molecule and its hydrogen-bonding pattern. The water molecules could be divided into different groups, according to their relative mobility. Some of the high-mobility water molecules were found to move as quickly as in the bulk phase, and they moved in to occupy the space vacated by the leaving retinol. The lowmobility water molecules, on the other hand, usually exhibited non-directional movement, and some of them were effectively exhibiting random motion ${ }^{36}$.

\subsection{Hydrophobic interaction}

The hydration properties of retinol as it emerged from its binding site were examined $^{37}$. $5.5 \AA$ was defined as the first hydrophobic hydration shell limit distance. When the ligand was bound, the number of water molecules within the first hydration shell of retinol was $44.7 \pm 0.5$, but this number increased to $76.2 \pm 1.4$ for the last $50 \mathrm{ps}$, when the ligand was unbound.

Morever, I identified a group of water molecules that were in the bulk region when retinol was bound, but came to coat retinol when the ligand had unbound. I examined the properties of these water molecules, and showed that they were indeed in the bulk phase in the beginning of the simulation, but came to adopt a hydrophobic-hydration configuration with respect to retinol towards the end of the simulation ${ }^{38}$.

\footnotetext{
${ }^{35}$ P.-L. Chau (2004) Water movement during ligand unbinding from receptor site, Biophysical Journal, 87, 121-128.

${ }^{36}$ see figures $8-10$ of reference 35

${ }^{37}$ reference 23

${ }^{38}$ reference 23
} 


\section{Modified Mutual Repulsion}

\subsection{Methods}

The mutual repulsion method was applied to unbind 5-hydroxytryptamine (5-HT) from a homology model of the $5-\mathrm{HT}_{3 \mathrm{~A}}$ receptor. The structure of the receptor was obtained from previous work ${ }^{39}$, and its structure is shown in figure 5 . Two different ligands, 5-HT and granisetron, whose chemical diagrams are shown in figure 6, are docked into one of the five binding sites of the receptor. 5-HT is an agonist of the receptor, and granisetron is an antagonist. Figure 7 shows one 5-HT molecule docked to the $5-\mathrm{HT}_{3 \mathrm{~A}}$ receptor.

The CHARMm22 potential was used throughout ${ }^{40}$. Two series of simulations were carried out. The first one consists of 5-HT docked into the binding site ${ }^{41}$, and the other with granisetron docked into the same site ${ }^{42}$. All simulations were carried out in vacuum.

In each simulation, the structure of the protein-ligand complex was minimised for 20000 steps using the zero-K minimisation method. All non-hydrogen atoms of the ligand, and all $\mathrm{C}_{\alpha}$ atoms of the protein were tethered to their original positions, and the structure heated up to $310 \mathrm{~K}$ over $250 \mathrm{ps}$, followed by an equilibration period of 100 ps. Data production was carried out after equilibration, with configuration dumping taking place every 1 ps. The time-step used was always $1 \mathrm{fs}$. A Nosé-Hoover thermostat was applied with a thermostat constant of 0.1 ps.

The unbinding required special care. Indeed, these simulations revealed a question with this unbinding method: mutual repulsion acted on the centre of mass of the two molecules, so if the unbinding trajectory was not around the line joining the two centres of mass, would the ligand leave the binding site? Initially, I implemented the unmodified mutual repulsion method, with the line of

\footnotetext{
${ }^{39}$ D.C. Reeves, M.F.R. Sayed, P.-L. Chau, K.L. Price and S.C.R. Lummis (2003) Identification of $5-\mathrm{HT}_{3}$ receptor agonist-binding residues using homology modelling, Biophysical Journal, 84, $2338-2344$.

${ }^{40}$ A.D. MacKerell et al. (1998) All-atom empirical potential for molecular modeling and dynamics Studies of proteins, Journal of Physical Chemistry B, 102, 3586-3616.

${ }^{41}$ structure 4 from D.C. Reeves, M.F.R. Sayed, P.-L. Chau, K.L. Price and S.C.R. Lummis (2003) Identification of $5-\mathrm{HT}_{3}$ receptor agonist-binding residues using homology modelling, Biophysical Journal, 84, 2338-2344.

${ }^{42}$ structure 2 from A.J. Thompson, K.L. Price, D.C. Reeves, S.L. Chan, P.-L. Chau and S.C.R. Lummis (2005) Locating an antagonist in the $5-\mathrm{HT}_{3}$ receptor binding site: a modelling and radioligand binding study, Journal of Biological Chemistry, 280, 20476-20482.
} 
force acting almost perpendicular to the membrane-wards direction. This forced the ligand out without any problems; there were no serious distortions of the protein structure. However, there was good evidence that the unbindning pathway faces membrane-wards (negative $z$-direction). The proposed trajectory was almost perpendicular to the line joining the centre of mass of the protein and the ligand (figure 8). It seemed more reasonable to force the ligand out along this direction. So instead of creating a force between the two centres of mass, I defined a dummy centre of mass for the receptor. This was placed in the positive $z$-direction of the ligand, so the mutual repulsive force would force the ligand out membranewards (blue arrow of figure 8). This, unfortunately would create a torque on the receptor, so the $\mathrm{C}_{\alpha}$ atoms of every 5 amino acids were tethered throughout the unbinding simulation.

In all simulations using the modified method, unbinding forces were increased to achieve an unbinding speed of about $20 \mathrm{~m} / \mathrm{s}$. In half the simulations, tethering remained on the $\mathrm{C}_{\alpha}$ atoms of every 5 amino acids, during the whole unbinding process (symmetric tethering). The amino acids within $5 \AA$ of the unbinding ligand were identified; these amino acids are called the "binding path amino acids". In the other half of simulations, the tethering was lifted from the $\mathrm{C}_{\alpha}$ atoms of these binding path amino acids (asymmetric tethering). In order to examine the effect of initial conditions on the unbinding trajectory, two starting conditions were used after equilibration. Either the unbinding simulation continued from equilibration (no velocity re-scaling), or the velocity of all particles were re-scaled to $310 \mathrm{~K}$ once, at the beginning of the data production run (velocity re-scaling) ${ }^{43}$.

\subsection{Results from 5-HT unbinding}

By examining the eight trajectories of unbinding, I identified key amino acids in the pathway ${ }^{44}$. A comparison with experimental data showed that many of the pathway amino acids were important for binding and function, and that mutation of those amino acids would reduce the binding and/or render the receptor

\footnotetext{
${ }^{43}$ A.J. Thompson, P.-L. Chau, S.L. Chan and S.C.R. Lummis (2006) Unbinding pathways of an agonist and an antagonist from the $5-\mathrm{HT}_{3}$ receptor, accepted for publication in the Biophysical Journal.

${ }^{44}$ see tables 1 and 2 of reference 43
} 
non-functional ${ }^{45}$. This work showed that even in the absence of aqueous solvent, molecular dynamics simulations were capable of giving us a qualitative understanding of intermolecular interaction in biological systems.

\subsection{Future directions}

This work shows that, under certain circumstances, the mutual repulsion method can allow the ligand to follow an unbinding trajectory very different from the line joining the centres of mass of the ligand and of the receptor. Whether this is generally applicable is not certain. A more useful method would be to unbind a ligand using random forces ${ }^{46}$.

A random force method has been developed by Lüdemann et al. ${ }^{47}$ In this method, a force is applied to the ligand in a random direction. If the ligand cannot travel a certain distance $r_{\min }$ within a defined time period, the path is abandoned and another direction chosen, until the ligand exits the binding site. The random force $\mathbf{F}_{\text {rand }}$ is expressed thus:

$$
\mathbf{F}_{\text {rand }}=k \mathbf{r}_{o}
$$

where $k$ is a force constant which is kept constant throughout the whole simulation, and $\mathbf{r}_{o}$ is the unit vector whose magnitude is also kept constant, but whose direction is changed randomly if the ligand velocity drops below a certain value.

In their implementation, Lüdemann et al. used a time-step of $2 \mathrm{fs}$, and for each random direction, between 10 to 100 steps were executed before deciding if a new random direction was required. Obviously the longer the trial period, the greater the danger of imposing conformational changes on the protein. They also maintained the unbinding speed of the ligand between $0.025 \mathrm{~m} / \mathrm{s}$ and $0.1 \mathrm{~m} / \mathrm{s}$.

As test systems, they produced 14 trajectories on the cytochrome P450camcamphor complex, 4 trajectories on the same protein complexed with 5-hydroxycamphor, and also 4 trajectories using the ligand endo-borneol allyl ether. They discovered three main exit pathways, which they named pathways 1 to 3 . Camphor

\footnotetext{
${ }^{45}$ reference 43

${ }^{46}$ private communication with Søren Toxværd in 1996.

${ }^{47}$ S.K. Lüdemann, V. Lounnas and R.C. Wade (2000) How do substrates enter and products exit the buried active site of cytochrome P450cam? 1. Random expulsion molecular dynamics investigation of ligand access channels and mechanisms, Journal of Molecular Biology, 303, 797-811.
} 
showed a small preference in the order of pathway $2>$ pathway $1>$ pathway 3 , whilst 5-hydroxy-camphor and endo-borneol allyl ether only existed via pathway 1 or 2 , with endo-borneol allyl ether showing a preference for pathway 1 , and 5hydroxy-camphor having equal preference for the two possibilities. Interestingly, the pathway with the lowest $B$-factor is the one least commonly used (pathway $3)$.

Unfortunately, the random force method developed is not linked to any free energy estimation routine, so would be able to predict an unbinding pathway only. Work is in progress to couple the two methods.

\section{Conclusions}

Since the advent of simulations methods in the 1950's, they have been gradually taken up by biologists to study ligand-receptor interactions. This method has proved to be another useful tool in the armamentarium of scientists. Used properly, it is capable of giving us information which complements experimental data.

DL_POLY has been used for some of the simulations described. It has many advantages. It is a well-written and transparent piece of software based on rigorous physics (for example, the Nosé-Hoover thermostat ${ }^{22}$ available gives an NVT ensemble, unlike some temperature-stabilising regimes which makes the temperature constant but does not give an NVT ensemble $\left.{ }^{48}\right)$. The intrinsic options available caters for a large variety of potentials and simulation conditions, it is parallelisable, and the programme package allows the user to alter the source code easily; this is important in research where new potentials, simulation conditions and/or simulation methods need to be incorporated.

The main problem related to using DL_POLY is to generate the CONFIG and FIELD files used by DL_POLY from the coordinates of a biomolecule, usually in the Protein DataBank (PDB) format. A related piece of software, DL_PROTEIN, contains a suite of programmes called build-topology to perform this. Unfortunately, these programmes are rather unwieldy, and, at the time of my carrying out

\footnotetext{
${ }^{48}$ H.J.C. Berendsen, J.P.M. Postma, W.F. van Gunsteren, A. DiNola and J.R. Haak (1984) Molecular dynamics with coupling to an external bath, Journal of Chemical Physics, 81, 36843690 .
} 
the retinol simulation work, ridden with bugs. I had to locate and correct them. I note that DL_POLY is not yet widely used by biological scientists, which is a pity because it is a more versatile and scientifically-rigorous piece of software than many others. A better and more user-friendly interface between the PDB format and the DL_POLY CONFIG and FIELD files should encourage more biological scientists to switch to this package.

\section{Acknowledgements}

I am grateful to Ole Olsen for the original idea of mutual repulsion, Søren Toxværd, Andrew Hardwick, Ruth Lynden-Bell, Chan Shek Ling, Owen Saxton, Alex Selby, André Juffer, Peter Howe, Wolfgang Rieping and Michael Witty for mathematical discussions, Bill Smith for advice on implementing mutual repulsion on DL_POLY, Simone Melchionne for suggesting the method of adiabatic switching, and Greg McMullan, Jenny Barna, Nick Maclaren, Guy Cormier and Leung Hin-Tak for technical help. I thank the UK Biotechnology and Biological Sciences Research Council for the award of a David Phillips Fellowship, New Hall, Cambridge, for a Research Fellowship, and the Royal Society for the award of a European Science Exchange Programme travel grant, for different parts of this work. Provision of computational resources on the Hitachi SR 2201 at the High Performance Computing Facility of the University of Cambridge is gratefully acknowledged. 


\section{Figures}

Figure 1: Diagram showing the structure of the bovine serum retinol-binding protein (1HBP), at the beginning of the unbinding simulation.

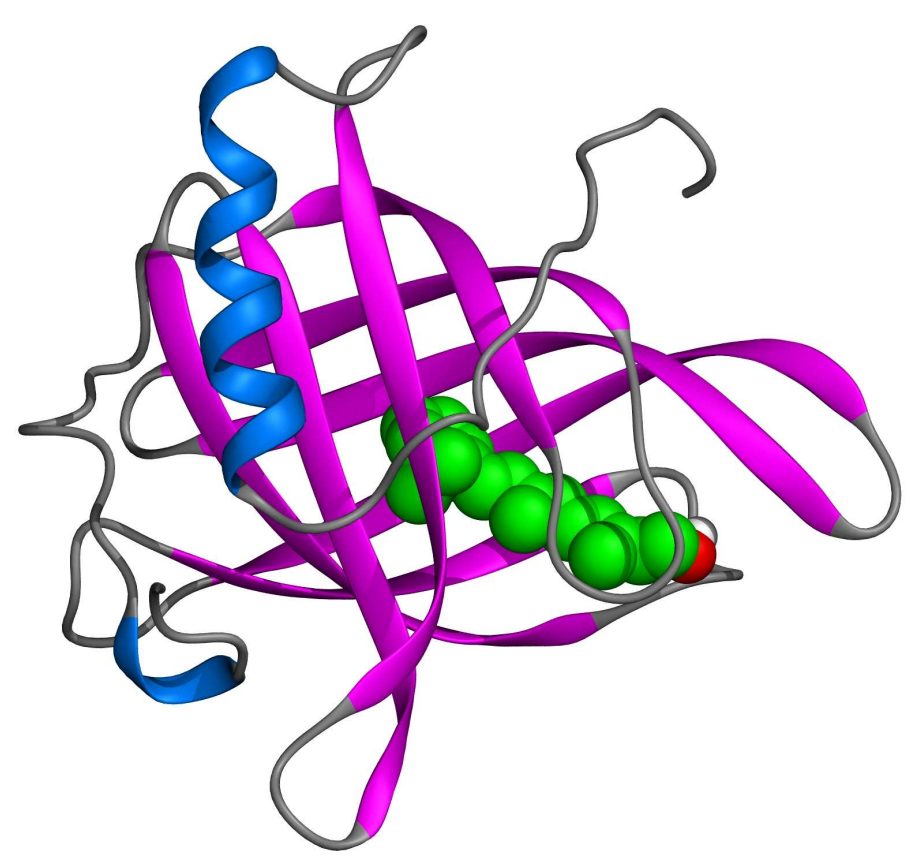


Figure 2: Diagram showing the structure of the bovine serum retinol-binding protein (1HBP), towards the end of the simulation.

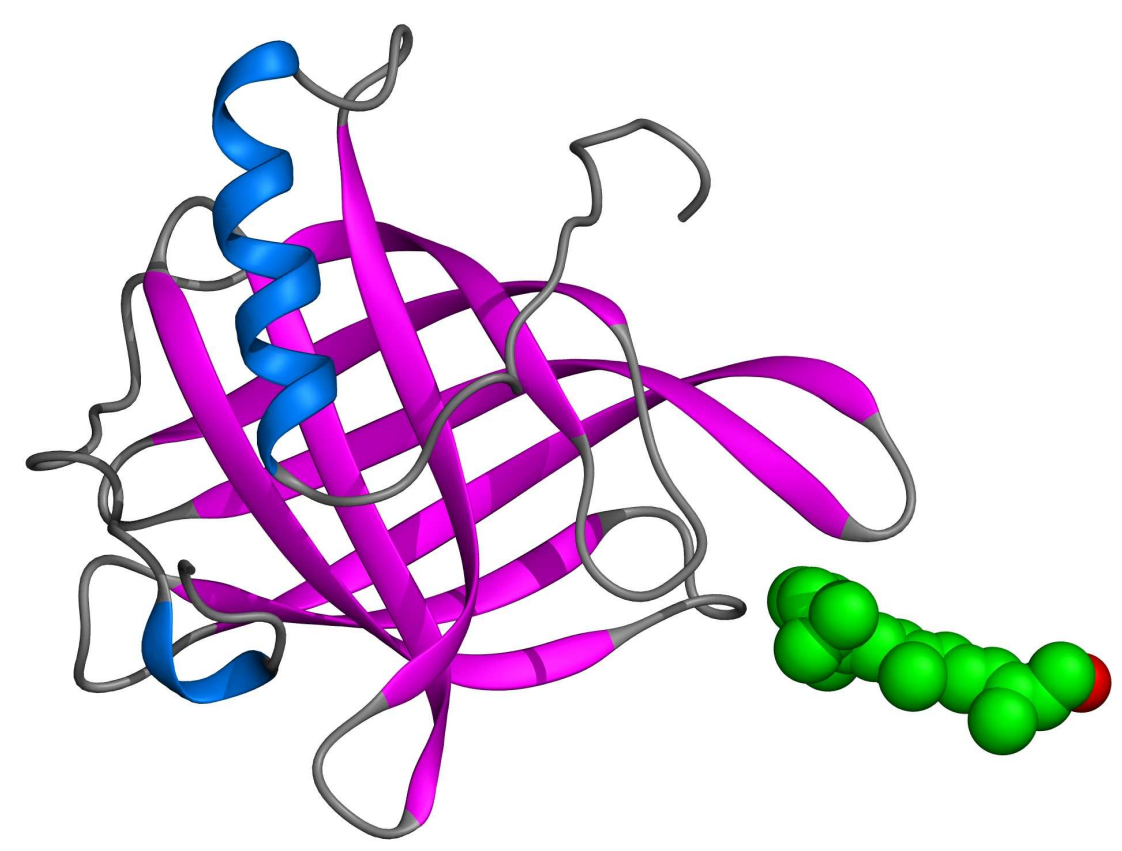


Figure 3: Diagram showing the volume of the binding site during the course of unbinding.

\section{binding site volume during unbinding}

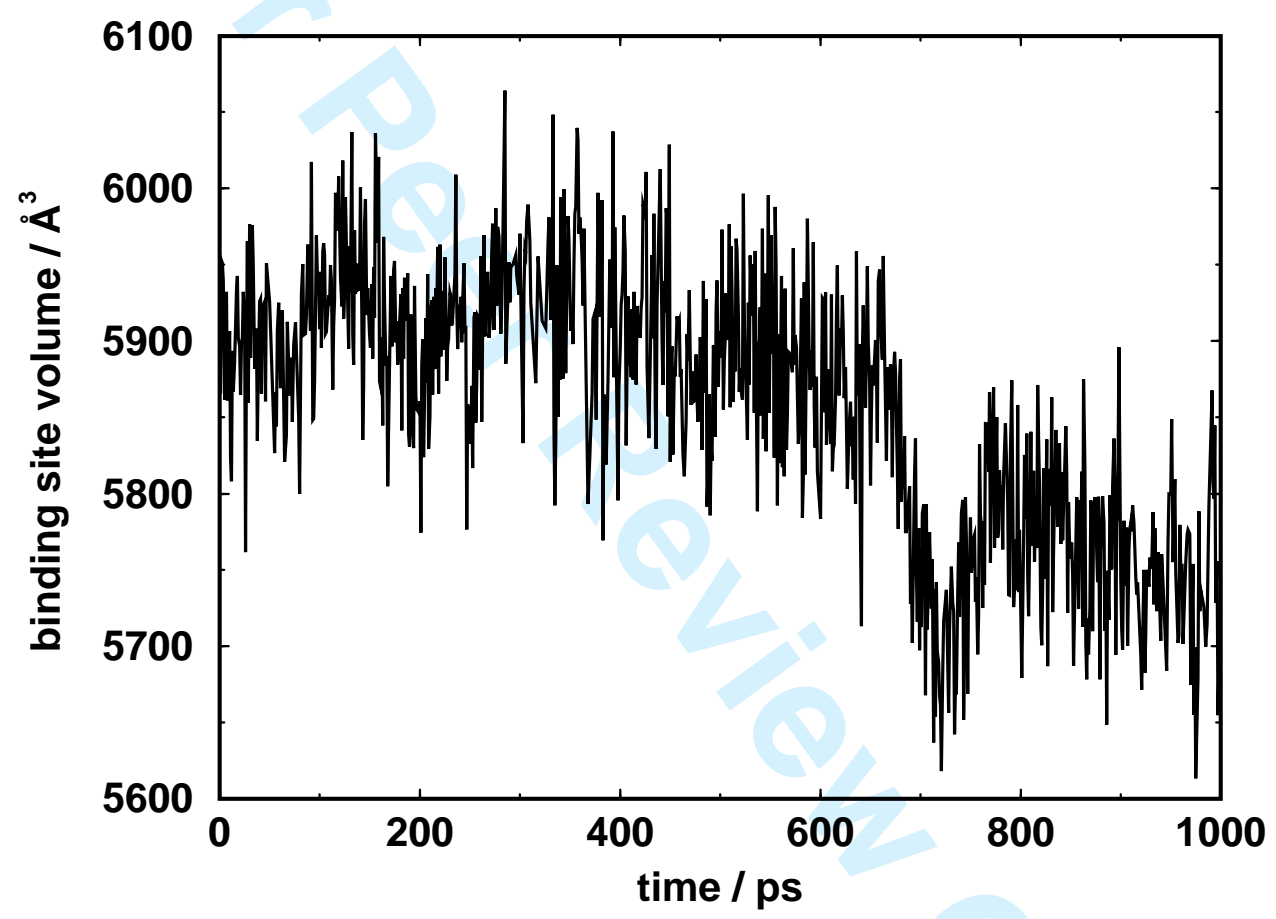


Figure 4: Diagram showing the number of water molecules inside the binding site during the course of unbinding.

\section{number of water molecules in binding site}

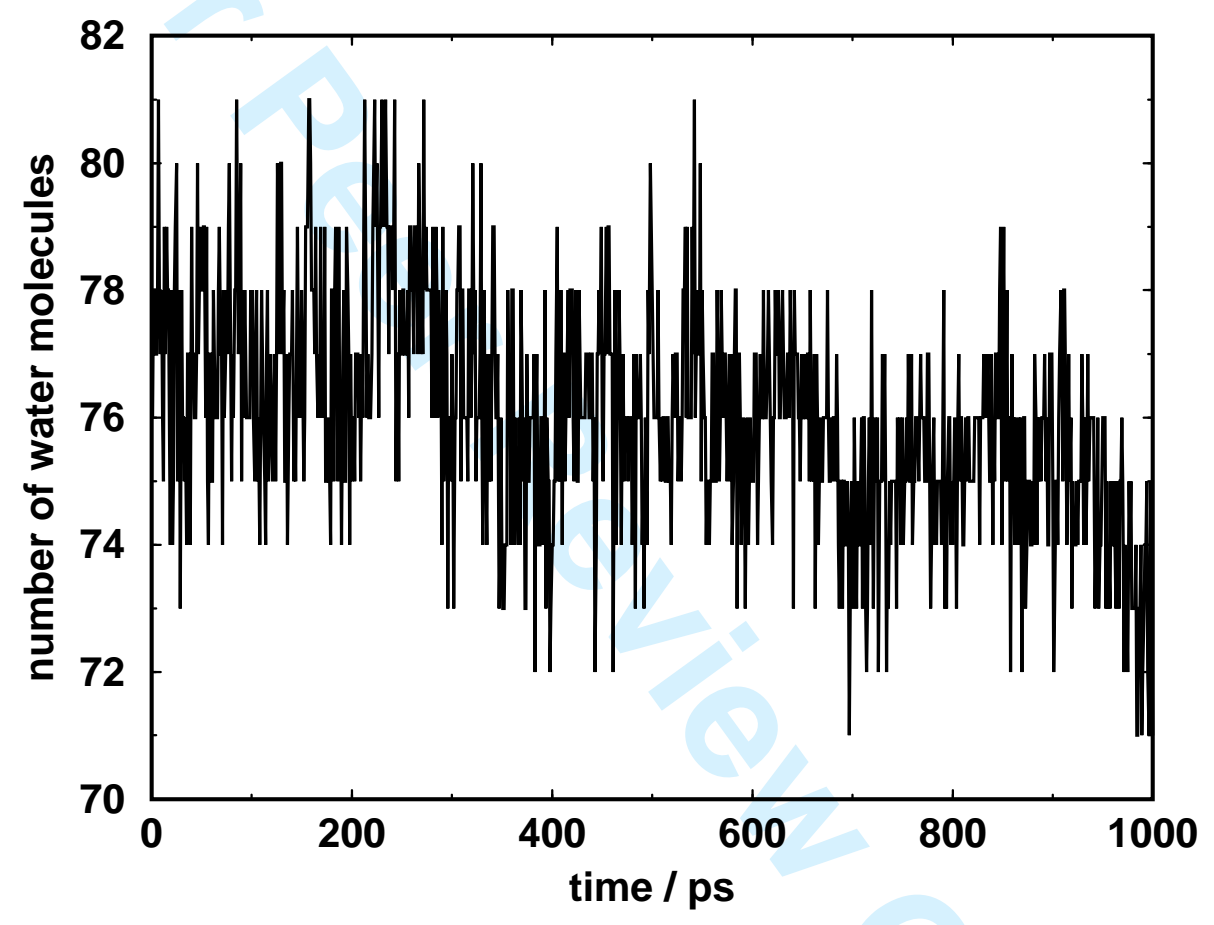


Figure 5: Diagrams showing the structure of the homology model of the extracellular domain of the $5-\mathrm{HT}_{3 \mathrm{~A}}$ receptor. (a) view from the extracellular space towards the membrane. The central pore leads to the ion channel; (b) side view of the extracellular domain. The membrane is at the bottom, and the extracellular space at the top. Each subunit is coloured differently, and the binding site is at the interface of two subunits.
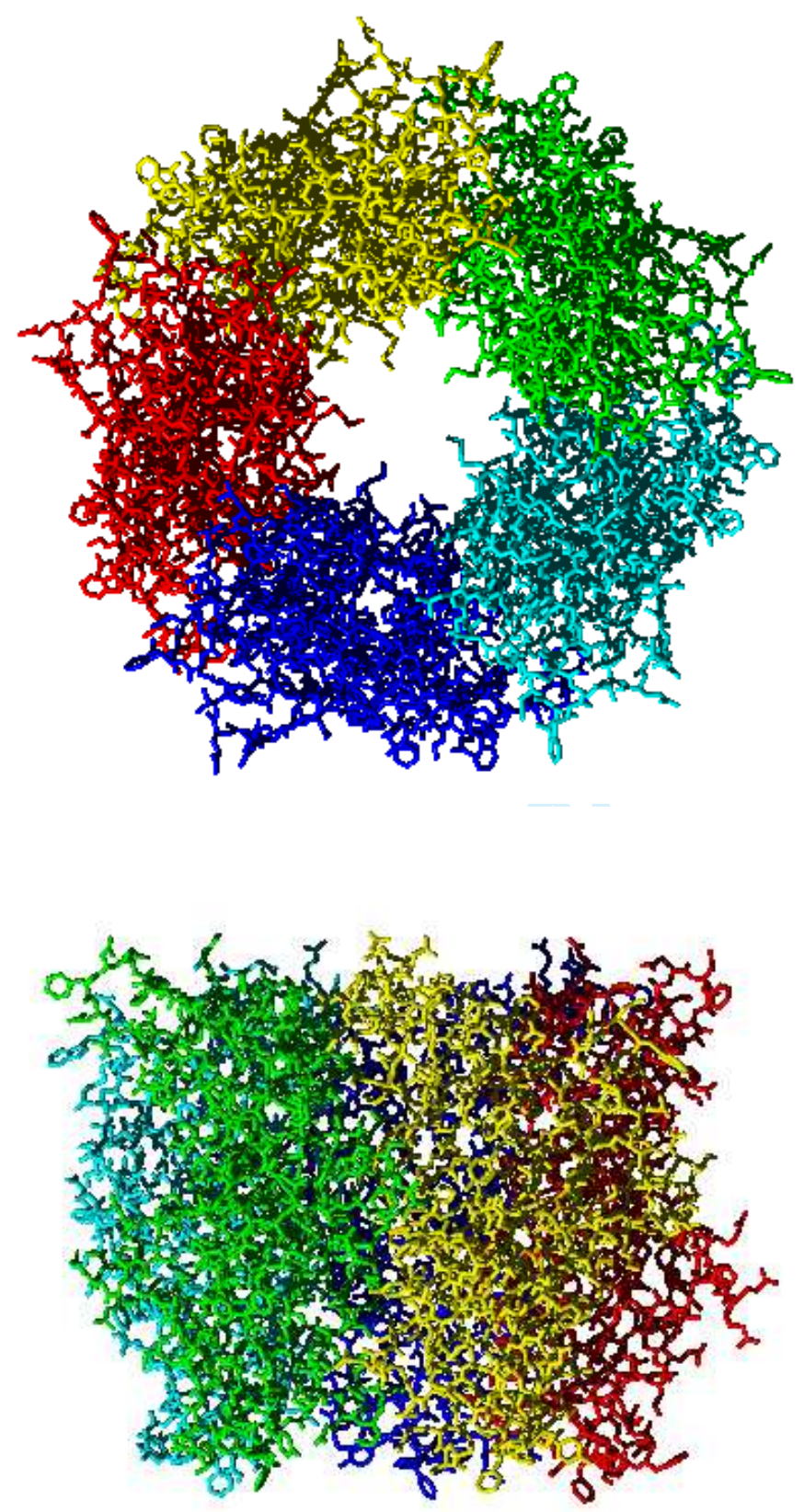
Figure 6: Chemical structures of (a) 5-HT and (b) granisetron. The hydrogenbonding groups are in red.
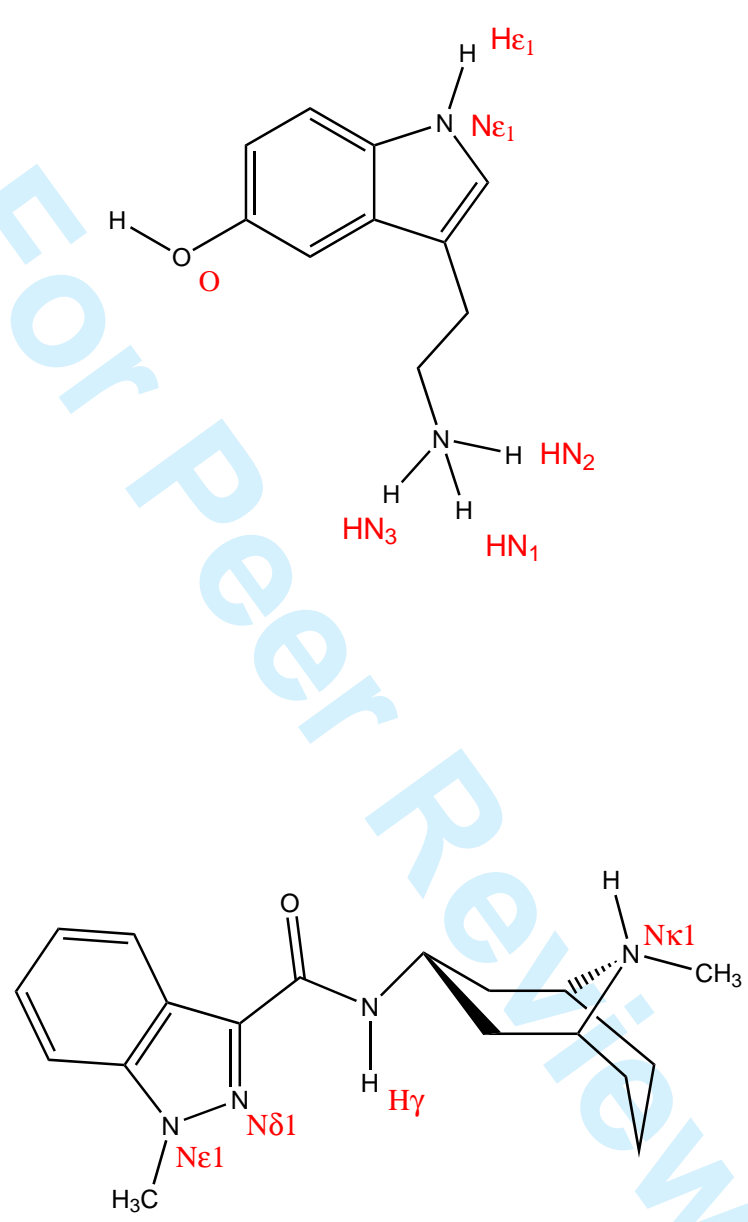
Figure 7: Diagram showing 5-HT docked to its receptor, binding to the interface between two subunits. The protein is colour-coded according to subunit. The drug is displayed in CPK model.

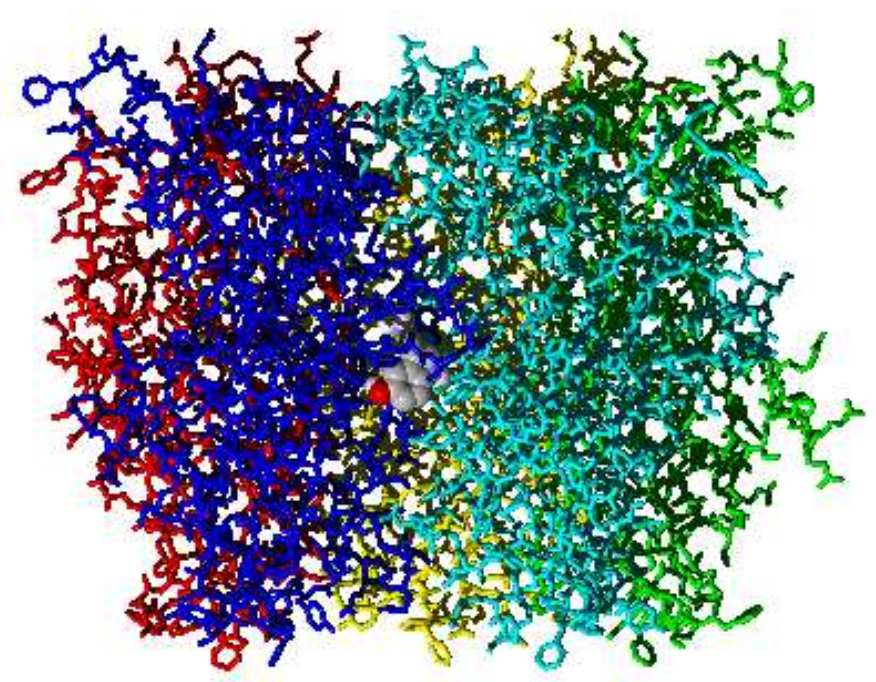


Figure 8: Diagram showing the relative disposition of the centre of the receptor (magenta circle) and the ligand (shown in space-filling model). The protein receptor is shown in grey wireframe. A mutually repulsive force would have forced the ligand out along the magenta arrow. However, there is evidence to show that the ligand most probably comes out membrane-wards, along the direction of the blue arrow. A dummy push-point was thus placed on the extracellular side of the protein to push the ligand out in the membrane-wards direction.

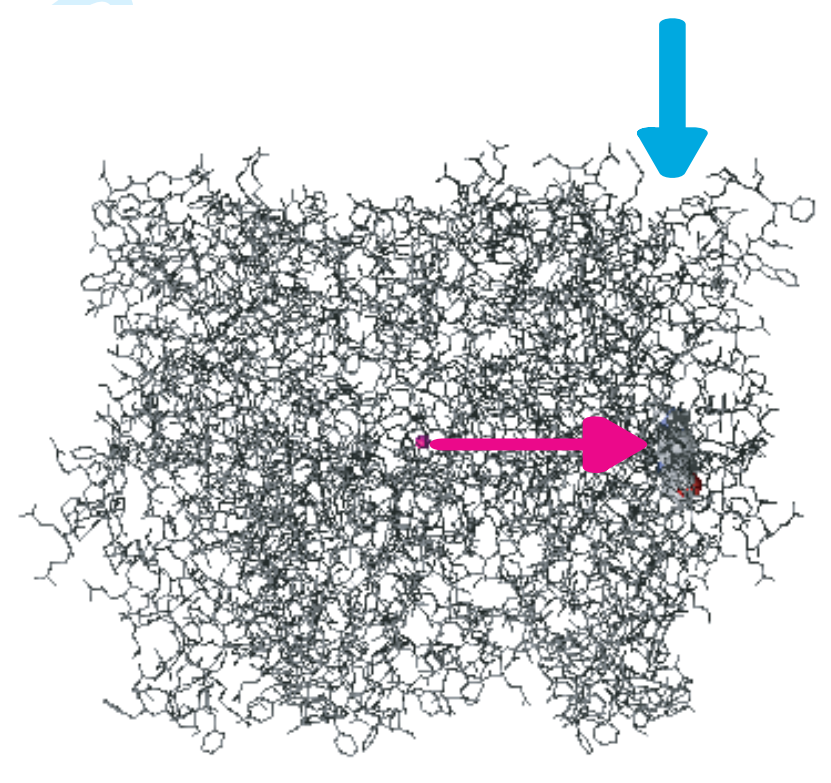

